\title{
Minimization of Information Asymmetry Interference using Partially Overlapping Channel Allocation in Wireless Mesh Networks
}

\author{
Sadiq Shah ${ }^{1}$ \\ Department of \\ Computer Science, \\ FATA University \\ FR, Kohat, Pakistan
}

\author{
Khalid Saeed ${ }^{2}$ \\ Departement of Computer Science \\ Shaheed Benazir Bhutto \\ University, Sheringal, Dir-Upper Pakistan
}

\author{
Mustafa Khan ${ }^{3}$, Rafi Ullah Khan ${ }^{4}$, \\ Mohib Ullah Khan ${ }^{5}$, Misbah Daud ${ }^{6}$, \\ Arbab Waseem Abbas ${ }^{7}$ \\ The University of \\ Agriculture, Peshawar, Pakistan
}

\begin{abstract}
Wireless Mesh Network (WMN) is a developing technology that has a great impact on the improvement of the performance, flexibility and reliability over the traditional wireless networks. Using multi-hop communication facility these networks are installed as a solution to extend last-mile access to the Internet. WMN has already been deployed but still it faces certain issues regarding channel assignment and interference. One of the well-known interference issues is Information Asymmetry (IA) interface that results in increased retransmission ratio, end-to-end delay, and thus decreases the overall network capacity of WMN. Various studies have been done in the past to minimize information asymmetry interference using limited number of orthogonal or non-overlapping channels i.e. 1, 6 and 11 from IEEE 802.11b radio technology. In recent studies, it is mentioned that partially overlapping channels called POCs can be used to maximize network capacity. The purpose of this research is to minimize Information Asymmetry (IA) interference problem by proposing a channel assignment model called Optimal Partially Overlapping Channel Assignment (OPOCA). In this research, comparison has been made between OPOCA and existing Information Asymmetry Minimization (IAM) model. Through extensive simulations it has been verified that the proposed OPOCA model gives $8 \%$ better results as compared to existing IAM model.
\end{abstract}

Keywords-Wireless Mesh Network (WMN); information asymmetry; Optimal Partially Overlapping Channel Assignment (OPOCA); NOC; Information Asymmetry Minimization (IAM) model

\section{INTRODUCTION}

\section{A. Wireless Mesh Network}

Wireless Mesh Network (WMN) is a well-known technology that has the capability of better performance regarding flexibility and reliability from that of conventional wireless networks. WMNs have self-healing capabilities and are easier to install. Fixed wireless broadband networks of next generation are being installed increasingly as mesh networks, for the purpose to extend access and provide Internet globally. The wireless networks could be set up between hundreds of wireless mesh nodes that would talk to each other for the purpose to share the data through network across vast area. Nodes of wireless mesh network are small radio transmitters that acts like router (wireless) using current standards of WiFi (802.11 a, b and g) for the purpose of communications. A

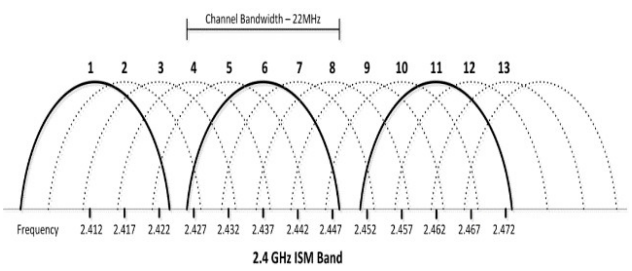

Fig. 1. Non-overlapping and Partially Overlapping Channels (POC) [3].

Wireless Mesh Network contains gateways, mesh clients and mesh routers. Mesh routers forward traffic to the gateways and from the gateways that might connect to the Internet, while the wireless devices, cell phones and laptops are referred as mesh clients [1]. Traditionally the wireless networks were supplied with only one radio interface. Though, the only interface intrinsically limits the overall network using only a single channel. In case of two neighboring links that are operating on the similar channel and transmit data concurrently, then the chances of interference is definitely more with each other. Multi-radio multi-channel (MRMC) systems, the nodes can receive and transmit simultaneous transmission to multiple neighbors can be easily done.

\section{B. Channel Assignment in WMNs}

The Channel Assignment (CA) in a multi-radio multichannel WMN comprises a mission to allocate channels to different radio interfaces in such a technique that achievement of effective channel utilization and minimization of the interference could be made possible. The Wi-Fi standard $802.11 \mathrm{~b} / \mathrm{g}$ works in $2.4 \mathrm{GHz}$ frequency spectrum and has supported transmission capacity of $11 \mathrm{Mbps}$. In IEEE $802.11 \mathrm{~b}$ spectrum 11 POC channels are used [2]. Those wireless channels that have spectrum overlap with the other working channels are referred as Partially Overlapped Channels (POCs). The NonOverlapped Channels (NOCs) are those channels that have no spectrum overlap with any other channels that are working. In IEEE $802.11 \mathrm{~b} / \mathrm{g}$ wireless standard which is quite well known, the largest channel that is orthogonal (non-overlapping) set contains channel 1, 6 and 11. One of foremost problems in proposing effective schemes of channel assignment using POCs is the adjacent channel interference that is the interference among two neighbors adjacent. In Fig. 1 IEEE $2.4 \mathrm{GHz}$ 


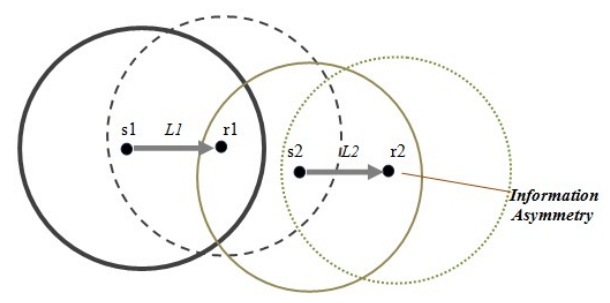

Fig. 2. Information Asymmetry (IA) interference in WMN.

spectrum is shown.

\section{Information Asymmetry Interference in WMN}

Interference plays a vital role when the number of different clients shares frequency using multiple channels. The interference in WMN is categorized as the coordinated and Information Asymmetry (IA) interference. Fig. 2 shows IA interference scenario. In case of IA the source nodes s1 and s2 are outer from the carrier sensing range (CS) of each other and the destination nodes $\mathrm{r} 1$ and $\mathrm{r} 2$ are also outer from the range of each others carrier sensing range CS. In the same way r2 and $\mathrm{s} 1$ are also located outside CS range of each other but $\mathrm{r} 1$ and s2 are inside the CS ranges of each other. In interference of Information Asymmetry (IA), the sender node is exterior to the Carrier Sensing range (CS) i.e., if there are two links L1 $(\mathrm{r} 1, \mathrm{~s} 1$,$) and L3 (r2, s2,) that are working on similar channel$ then following condition needs to be accomplished for IA interference [3].

$$
\begin{array}{ll}
\text { - } & \mathrm{d}(\mathrm{s} 1, \mathrm{~s} 2)>\mathrm{CS} \\
\text { - } & \mathrm{d}(\mathrm{r} 1, \mathrm{~s} 2)<\mathrm{CS} \\
\text { - } & \mathrm{d}(\mathrm{s} 1, \mathrm{r} 2)>\mathrm{CS}
\end{array}
$$

Here $\mathrm{d}$ means the distance between physical nodes and CS shows the Carrier Sensing range. In Fig. 2, the carrier sensing range of the sender nodes is represented by solid line circle and the circle with dotted line shows CS range of the receiving nodes.

\section{Paper Contributions}

To identify IA interference links in MR-MC wireless mesh network. To propose the most optimal channel assignment strategy in MR-MC WMN that will minimize IA interference effect and maximize network capacity in MRMC wireless mesh network. To compare the proposed model results with existing model called Information Asymmetry Minimization (IAM) model using IEEE $802.11 \mathrm{~b}$ technology. To verify proposed model results using extensive simulations.

\section{RELATED WORK}

Authors in [4] examined the decline in capacity of multiple radios WMN because of interference the links of communication through wireless network. This method was fundamentally used to reduce the interference among different inter communication links and maximize the network capacity. CA approach which is based on cluster is installed in order to lessen the complication of CA and use again the channel in the complete different cluster. Similarly [5] proposed that the efficient use of available channels improves the overall performance of a system. Most of the channels are partial overlapped. It is not always unsafe to use the partially overlapped channels. Different radio spectrum is discussed. The author gives overview of different interference constraints that are hard constraint, soft constraint, traffic demand constraint and orthogonal constraint. This paper gives the solution to prevent interference occurred due to partially overlapping.

A detailed survey was presented in [6] regarding survey of some of the channel assignment approaches. In this research the network model and key design concerns are identified. The comparisons of four graph based algorithms have been made that are Breadth First Search Channel Assignment (BFS-CA), Minimum Interference Survivable Topology Control (INSTC), Connected Low Interference Channel Assignment (CLICA), Centralized Tabu-based Algorithm (CTA). According to [7] the solid information about issues concerned to the most favorable usage of radio channels and interfaces in WMNsis examined. The aim of this paper is to provide new central model of channel assignment that is called First Random Channel Assignment algorithm that is associated with the two other CA techniques called Load Aware Channel Assignment (LACA)and Clear Channel Assignment (CCA) by the equal Quality of Service parameters. NS-2 network simulator is used for simulation. In [4] the author compared the results of channel assignment for both dense and sparse MRMC WMN topologies through extensive simulation. The model optimization gives optimal outcomes for the environments where the non-coordinated interference is more that in sparse environments. An algebraic model is formulated that gives strategy of an optimized channel assignment. In this paper the simulation results showed that the intended optimization model performance is $19 \%$ better in sparse MRMC WMN topologies where the nCO interference is high [4].

Venkata et al., in [8] recommended two new channel assignment algorithms in this research, BFS-MinNI, BFSMaxNI to minimize the interference for WMN. The results were compared of both the proposed CA algorithm and the existed algorithms like BFS-CA and CCA. The co-located interference between the radio interfaces and measure the degree of interference value of a variety of grid sizes and evaluated the performance of different algorithms. The experiment based on BFS-MinNICA results in significant performance improvement at varying interference levels. Wang et al., in [9] proposed that the network capacity can be improved by utilizing POCs, which assign channel to all the links while minimizing total network interference. The direct relationship between interference range and channel separation was calculated. The author evaluated that as the network traffic between the internet and clients become prevailing; distance from the gateway, the number of neighboring nodes and interference are used to resolve the channel assignment order of the links. The simulation results showed that the end to end delay and network throughput can be improved by making use of both non orthogonal channels as well as orthogonal channels.

Sadiq et al., [11] proposed an Information Asymmetry Minimization model called IAMin to minimize information asymmetry interference in multi-radio-multi-channel wireless mesh networks. The proposed channel assignment model optimally allocate IEEE $802.11 \mathrm{~b} / \mathrm{g}$ non-overlapping channels to wireless 
TABLE I. NOTATION USED IN MODEL

\begin{tabular}{|c||c|}
\hline SYMBOL & DESCRIPTION \\
\hline $\mathrm{L}$ & Set of all mesh links \\
\hline $\mathrm{H}$ & Number of channels that is 11 \\
\hline$\lambda$ & Traffic flow fraction on any link \\
\hline$e_{i}$ & A WMN link \\
\hline$c_{j}$ & Frequency channel \\
\hline $\mathrm{k}$ & The set of non-overlapping channels that is 3 \\
\hline $\mathrm{C} c_{j}$ & frequency channel capacity \\
\hline $\mathrm{x}\left(e_{i}, c_{j}\right)$ & Channel $c_{j}$ assigned to link $e_{i}$ \\
\hline $\mathrm{IA}\left(e_{i}\right)$ & Set of IA interfering links of link $e_{i}$ \\
\hline $\mathrm{CO}\left(e_{i}\right)$ & Coordinated interference link of $e_{i}$ \\
\hline
\end{tabular}

links of multi-radio multi-channel wireless mesh network. The optimal channel assignment not only minimizes information asymmetry problem, but also maximizes the overall network capacity. Simulation result show that the proposed optimization model successfully minimizes information asymmetry interference and maximizes the capacity in sparse scenarios of multiradio multi-channel wireless mesh networks up to 8

Another research article [12], compares near-hidden and information asymmetry problems in wireless mesh networks. The comparison between existing Optimal Channel Assignment Model (OCAM) and Information Asymmetry and Near Hidden Minimization (INM) model is done to find the networks capacity.The proposed model considers three nonoverlapping channels 1,6 and 11 from IEEE802.11b standard.An extensive simulation in OPNET shows that the proposed INM model performs $7 \%$ better than the existing OCAM model.

\section{RESEARCH Methodology}

In this section the optimization model based on partially overlapping channel assignment is proposed to minimize Information Asymmetry (IA) interference problem.

\section{A. Proposing Linear Programming POC model}

A linear programming model is proposed that is base on Partially Overlapping Channel (POC) assignment that consists of objective function and several channel assignment constraints. The wireless mesh network allows simultaneous use of multiple channels to increase the aggregate capacity. Partially Overlapped Channels has the potential of increasing the capacity in WMNs by allowing more links to transmit at the same time. Channel assignment using POC allows significantly more flexibility in sharing the wireless spectrum. Here POC model is implemented for maximizing the network throughput and performance using maximum channel resources in a multichannel multi-radio wireless mesh network.

\section{B. Problem Formulation}

In this section we formulate a linear optimization model. The model comprises of decision variable, objective function and certain set of constraints. Table I shows all the symbols and notations used in this model.

\section{Existing Model}

The IEEE 802.11b standard is used in existing IAMin model. Each networks node is well-equipped with two or more radio interfaces for taking advantage of multi-radio multichannels WMN. The existing model is Information Asymmetry Minimization (IAMin) model. This model consist a set of definite constraints, objective function and decision variable.

1) Decision Variable : In the decision variable model an IEEE $802.11 \mathrm{~b}$ non overlapping channel that is $c_{j}$ is allocated to a link named as $e_{i}$. It is stated that if any link that is directed $e_{i}$ operates on any channel $c_{j}$ in a network then it equates 1 and if no channel is assigned to any specified link then it equates 0 . Such type of a variable (usually decision variable) is also known as binary variable [4].

$$
x\left(e_{i}, c_{j}\right)=\left\{\begin{array}{l}
1 \text { Directedlink } e_{i} \text { active on channel } c_{j} \\
0 \text { otherwise }
\end{array}\right.
$$

2) Set of Constraints: Constraints that are also the restrictions and limitations on an optimization model and they explain the unfavorable results. The interference effects between the links can be minimized for the improvement of the performance of overall network. Following are some set of constraints used for this channel assignment model.

- Individual channel per link constraint

The first constraint of the optimization model presented makes it sure that each and every link in a set of L (edges) should be allocated only one channel from the set $\mathrm{H}$. $\mathrm{H}$ is the set of total number of channels. Equation of single channel per link constraint affirms that if is a link that is assigned to a channel than calculation of overall channels evaluates to 1 [4].

$$
\sum c_{j} \in H x\left(e_{i}, c_{j}\right)=1 \quad \forall e_{i} \in L, c_{j} \in H
$$

- Channel Capacity Constraint

If the links that are coordinated are working on similar frequency channel then there is minimum interference and maximum network performance. The coordinated links are not affected by the interference. The capacity of a frequency channel is in fact dispensed amongst all the coordinated interfering links. The rate of traffic at a link should not go beyond the capacity of this link. The constraint that is given below indicates that the capacity of a channel is contributed when numerous corresponding links are active on a similar channel [10].

$$
\begin{gathered}
x\left(e_{i}, c_{j}\right) \cdot \lambda\left(e_{i}\right) \cdot f\left(e_{i}\right)+\sum_{e_{k} \in \epsilon_{c o}\left(e_{i}\right)} x\left(e_{k}, c_{j}\right) \cdot \lambda\left(e_{k}\right) \cdot f\left(e_{k}\right) \leq C c_{j} \\
\forall e_{i} \in L, c_{j} \in H
\end{gathered}
$$

- Information Asymmetry interference Constraints

The IA interference constraint shows that if there are multiple IA interfering links in a network then only one channel $c_{j}$ will be assigned to them. The equation shows that $e_{i}$ and $e_{k}$ are numerous links that are operating on the same channel i.e., $c_{j}$ in a network. Individual link will be active on channel $c_{j}$ in the interference region [10]. Fig. 3 shows one of the examples of IA interference.

$$
x\left(e_{i}, c_{j}\right)+\sum e_{k} \in I A(e i) x\left(e_{k}, c_{j}\right) \leq 1
$$




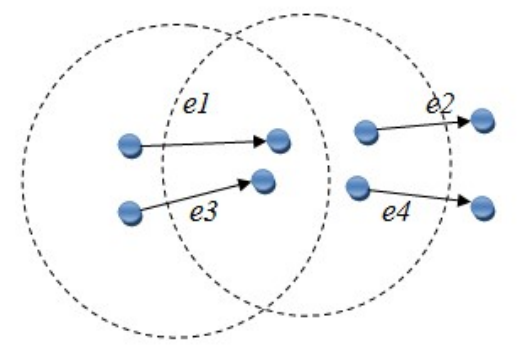

Fig. 3. Information Asymmetry links Fig. 3 illustrates information asymmetry links. The channel $c_{j}$ is assigned to different links that are e1,e2, e3 and e4. The sender nodes are outside each other CS.

$$
\forall e_{i} \in L, \forall c_{j} \in H, e_{k} \in L
$$

\section{Proposed Model}

The proposed model consist of node-radio constraint and Partially Overlapping Channel constraints. The proposed model is Optimization Partially Overlapping Channel Assignment (OPOCA) Model.

1) Node-Radio Constraint: Such type of constraint relates to nodes of wireless mesh network that comprises of multiple radios. A node in a network can use at most $n\left(v_{i}\right)$ radios in a given period of time for transmission or receiving of data or used for both. This leads towards the subsequent constraint. It is made sure by the constraint that the total quantity of channels that operates on various links of an incident node must not increase than the number of radio interfaces on that specified node [4].

$$
\begin{gathered}
\sum_{c_{\mathrm{j}} \in H} \sum_{e_{i} \in I\left(v_{i}\right)} x\left(e_{i}, c_{j}\right) \leq n\left(v_{i}\right) \\
\forall e_{i} \in L, \forall c_{j} \in H, \forall v_{i} \in V, c_{j} \in H, e_{i} \in L
\end{gathered}
$$

2) Partially Overlapping Channel (POC) Constraint: Partial overlap among different channels decreases the received flow transmission on one single channel if same channel is assigned to neighboring links. Two nodes can operate on the same channel as long as they do not interfere with each other. The minimization in the interference range increases with increase in separation. The channels, with complete reduction perceived over non-overlapping channels. In Fig. 4 POCs can be in use to interconnect to the number of networks or can be used to add flexibility to the routing infrastructure by creating additional edges in the mesh network topology. In Table II, the channel separation illustrates the difference in channel numbers which is denoted by $c s\left(c_{i}-c_{j}\right)$. In Fig. 4, there are two communication nodes pairs that are transmitting on a channel $c_{j}$ and channel $c_{i}$. The distance is increased gradually between these two nodes and interference range is recorded.

$$
\begin{gathered}
x(e i, c j)+\sum_{e k \in I A e i[c s|c i-c j|]} x(e k, c i) \leq 1 \\
\forall c i \in \operatorname{poc}(c j)
\end{gathered}
$$

TABLE II. CARRIER SENSING RANGES [3]

\begin{tabular}{|c||c|c|c|c|c|c|}
\hline Channel Separation(M) & 0 & 1 & 2 & 3 & 4 & 5 \\
\hline $\mathrm{i}=1$ & 13.26 & 9.08 & 7.59 & 4.69 & 3.21 & 0 \\
\hline $\mathrm{i}=6$ & 12.89 & 9.21 & 6.98 & 5.15 & 3.84 & 0 \\
\hline
\end{tabular}

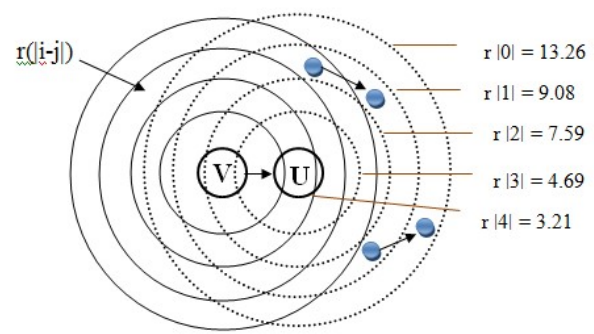

Fig. 4. Node $\mathrm{U}$ transmitting on $\mathrm{j}$ needs to be in cs-ci-cj-to interfere with node $\mathrm{V}$ transmitting on channel i [3].

\section{RESUlts AND Discussion}

In this section the results that are gathered from simulations are discussed.

\section{A. MATLAB WMN Topology}

In MATLAB three different topologies are created for experimental purposes. Each topology consists of 25 mesh nodes. The nodes are aligned close to each other, as the IA interference effect is verified on several numbers of nodes. The greatest Transmission range ( $\mathrm{Tr}$ ) of every node is 10 meters while the carrier sensing range (CS) is assumed to be 20 meters. The $\operatorname{Tr}$ is a range where successful communication is occurred among different nodes in the network. All nodes are vigorous and share information among each other.

Fig. 5 and 6 represents MRMC WMN topologies which are created in MATLAB software. In this figure the $\mathrm{x}$-coordinates and $\mathrm{y}$-coordinates are $80 \times 80$ meters. In the topologies solid lined circle signifies the CS range of a source node and dotted line circle denotes the CS of receiving node. The transmission range of the source node is represented by pink colored line circle. In Fig. 6 each topology consist of non-coordinated interference links and coordinated interfering links. In Fig. 5, $6,7,8$ and 9 , the carrier sensing range of WMN topologies is reduced gradually. Spectral Gap (Sgap) represents the carrier sensing range of a node in the network denoted as spectrum gap. In Fig. 5, sgap is taken as 0 which illustrates that it is the maximum carrier sensing range of a node. The IA links are identified for that topology. For link $(1,2)$ the identified IA links are $(3,4)(12,13)(13,14)$ and $(14,15)$. The coordinated links for the link $(1,2)$ are $(2,3)(3,4)(7,8)(16,17)(17,18)$ $(21,22)(22,23)(23,24)$. When the carrier sensing range is maximum, the IA interference will be more.

In Fig. 6 the value of Sgap is 1, it represents that the carrier sensing range reduce in sizes and reduced. In this case the IA link for $(1,2)$ is $(13,14)$ and the coordinated interfering links will remain the same. Further in Fig. 7 when sgap equals 2 the carrier sensing range minimizes and the IA links for $(1,2)$ are $(13,14)$. The Fig. 8 illustrates that when the sgap equals 3 than the identified IA links for node 1 and 2 are $(3,4)(12,13)$ respectively. In Fig. 9 the bare minimum 


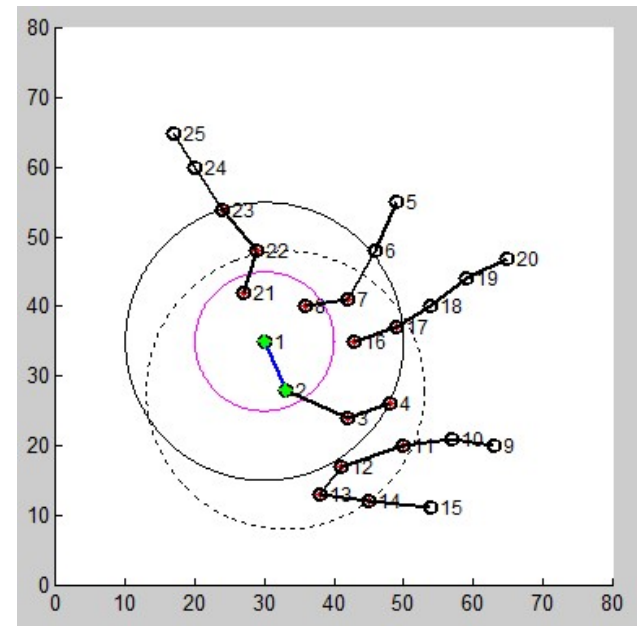

Fig. 5. WMN Topology for spectral gap 0 .

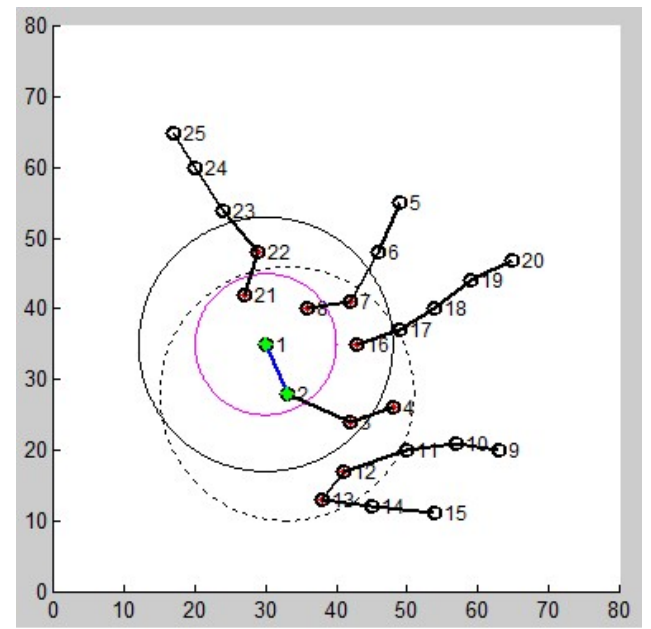

Fig. 6. WMN Topology for spectral gap 1.

carrier sensing range is acquired. Moreover when the value of sgap is equal to 4 the carrier sensing range excessively decreases and the IA interference link is $(3,4)$. This process is repeated for other topologies also. The outcome of information asymmetry interference is greater and the data rate will be highly decreased. In order to minimize IA interference between the nodes optimal channel assignment scheme is used.

Table III displays a list of coordinated interfering (CO) links and IA links. The first column shows the intended links, the second column represents coordinated interfering links. The third column represents Information Asymmetry interfering links. For identifying IA links each time the carrier sensing range is reduced. The sgap starts from value 0 and approaches to 4 . The value 0 represents maximum sensing range, the range decreases when the value reaches to 4 . The Carrier sensing range is reduced at sgap equals 4 . These links are derived from Fig. 5.

Fig. 10 and 11 represents two more topologies comprising of 25 nodes each. The terrain area has been taken $80 \times 80$ meters. The nodes alignment is based on x-axis and y-axis. In topology 10 and 11 four paths are taken. The black solid circle represents CS of source node and dotted circle represented

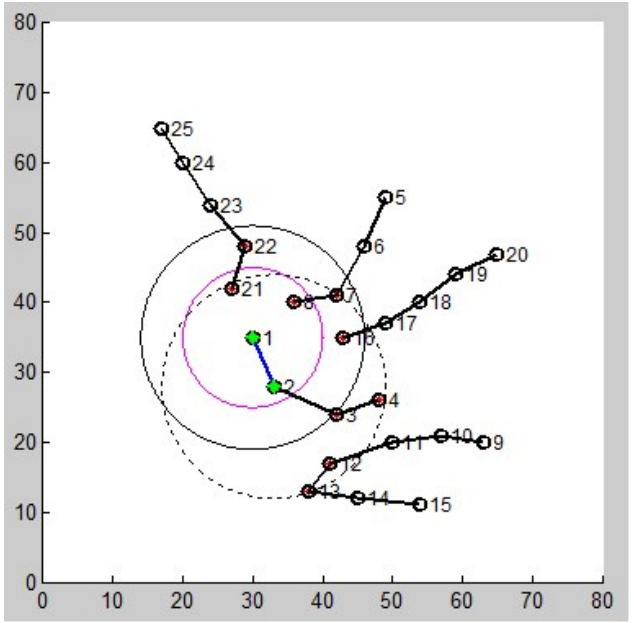

Fig. 7. WMN Topology for spectral gap 2.

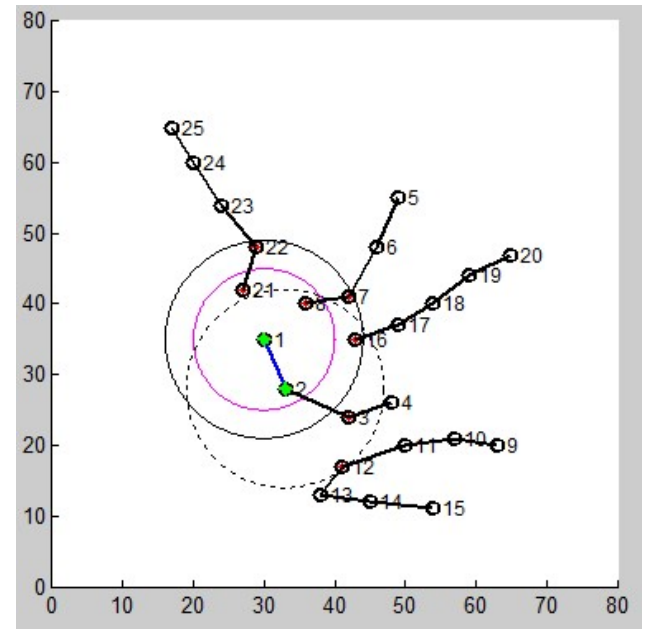

Fig. 8. WMN Topology for spectral gap 3.

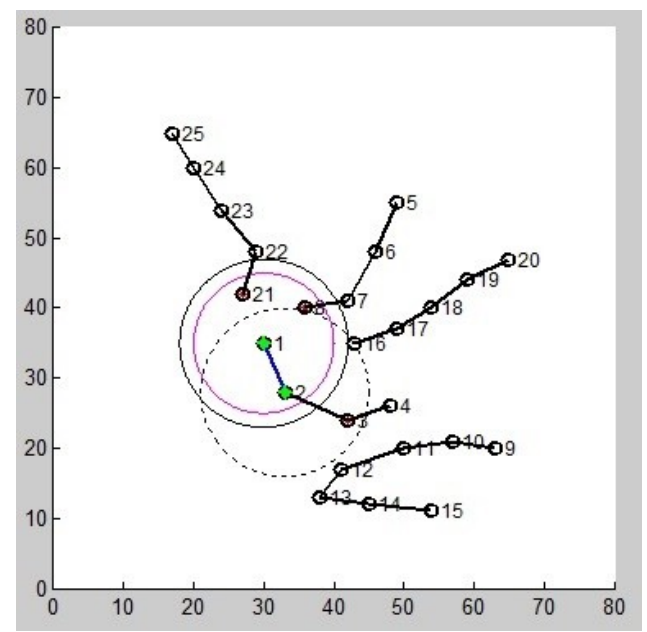

Fig. 9. WMN Topology for spectral gap 4.

CS range of destination node whereas pink circle represented transmission range of source. For both the topologies coordinated links and information asymmetry links have been 


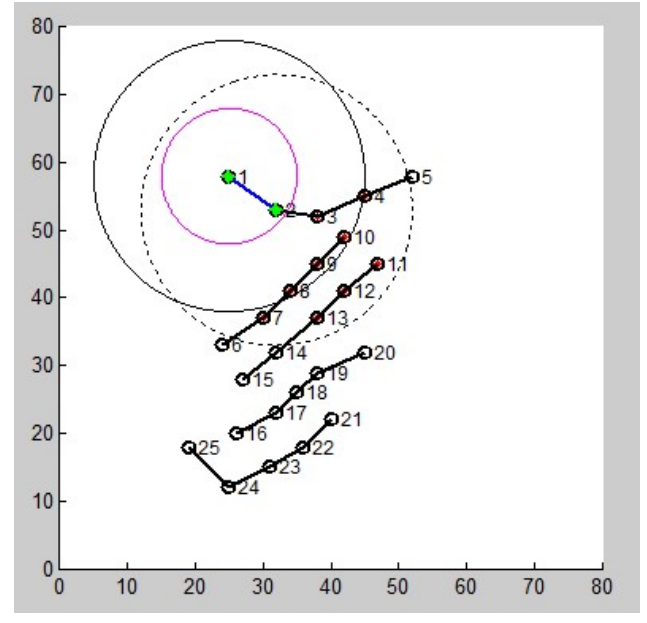

Fig. 10. MRMC-WMN MATLAB generated 25 node topology 1.

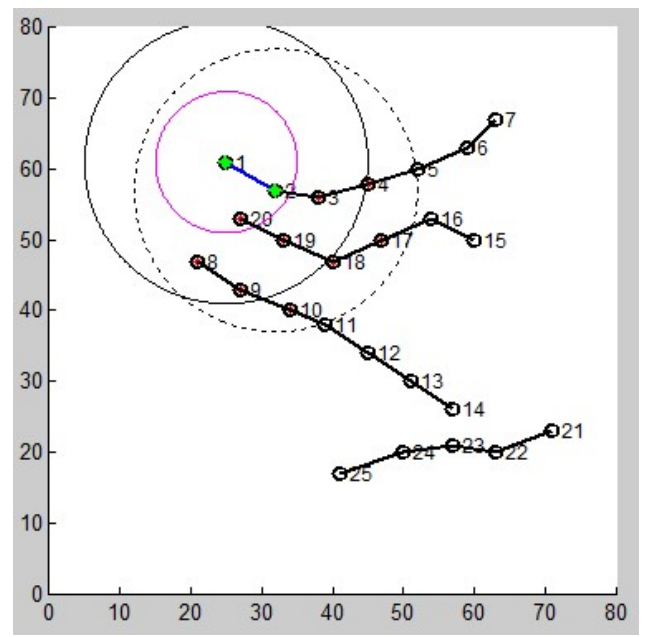

Fig. 11. MRMC-WMN MATLAB generated 25 node topology 2.

identified. The carrier sensing range is decreased by spectral gap value. The value of sgap ranges from 0 to 4 . At each sgap value the information asymmetry links are identified.

\section{B. Partially Overlapping Channel (POC) assignment in AMPL}

AMPL displays results regarding channel assignment. Partially Overlapping Channels (POCs) are assigned to the links in MRMC wireless mesh network. AMPL assign same traffic load to each link. That is varied from 50 to 500 packets/ sec. The spectrum IEEE $802.11 \mathrm{~b}$ has 11 channels. The partially overlapping channels are $1,2,3,4,5,6,7,8$, 9,10 and 11 have been considered for channel assignment. AMPL utilizes the gurobi solver for channel assignment results. The linear programming models have been executed. The file created is a text file that comprises of real execution of the proposed model. In gurobi solver the solve command gives the output or goal of the proposed model. The binary variable $\mathrm{xx}$ displays channel assignment and link. For instance $\mathrm{xx}\left[{ }^{*}, *, *\right]$ shows $\mathrm{xx}$ [souce link, destination link, channel assigned].

The variable $\mathrm{xx}[2,3,1]$ illustrates that channel 1 is allotted
TABLE III. LIST OF COORDINATED INTERFERENCE AND IA INTERFERENCE LINKS OF WMN TOPOLOGY

\begin{tabular}{|c|c|c|}
\hline LINK & COORDINATED LINKS & IA LINKS \\
\hline$(1,2)$ & $\begin{array}{c}(2,3)(3,4)(7,8)(16,17)(17,18) \\
(21,22)(22,23)(23,24)\end{array}$ & $\begin{array}{c}(3,4)(12,13) \\
(14,15)(13,14)\end{array}$ \\
\hline$(2,3)$ & $\begin{array}{c}(1,2)(3,4)(7,8)(11,12)(12,13)(13,14) \\
(14,15)(16,17)(17,18)(21,22) \\
\end{array}$ & $\begin{array}{l}(13,14)(14,15) \\
(16,17)(17,18)\end{array}$ \\
\hline$(3,4)$ & $\begin{array}{c}(1,2)(2,3)(7,8)(10,11)(11,12)(12,13) \\
(13,14)(14,15)(16,17)(17,18)\end{array}$ & $(17,18)(18,19)$ \\
\hline$(5,6)$ & $(6,7)(7,8)(17,18)(18,19)(19,20)$ & $\begin{array}{c}(7,8)(18,19) \\
(19,20)(22,23)\end{array}$ \\
\hline$(6,7)$ & $\begin{array}{c}(5,6)(7,8)(16,17)(17,18)(18,19) \\
(19,20)(21,22)(22,23) \\
\end{array}$ & $\begin{array}{c}(1,2)(2,3) \\
(22,23)\end{array}$ \\
\hline$(7,8)$ & $\begin{array}{c}(1,2)(2,3)(3,4)(5,6)(6,7)(16,17)(17,18) \\
(18,19)(19,20)(21,22)(22,23)\end{array}$ & $\begin{array}{c}(1,2)(2,3) \\
(22,23)(23,24)\end{array}$ \\
\hline$(9,10)$ & $(10,11)(11,12)(14,15)$ & $\begin{array}{l}(11,12)(12,13) \\
(17,18)(18,19) \\
\end{array}$ \\
\hline$(10,11)$ & $(3,4)(9,10)(11,12)(12,13)(14,15)(16,17)(17,18)(18,19)$ & $(12,13)$ \\
\hline$(11,12)$ & $(2,3)(3,4)(9,10)(10,11)(12,13)(13,14)(14,15)(16,17)(17,18)$ & Nil \\
\hline$(12,13)$ & $(2,3)(3,4)(10,11)(11,12)(13,14)(14,15)(16,17)$ & Nil \\
\hline$(13,14)$ & $(2,3)(3,4)(11,12)(12,13)(14,15)$ & Nil \\
\hline$(14,15)$ & $(2,3)(3,4)(9,10)(10,11)(11,12)(12,13)(13,14)$ & Nil \\
\hline$(16,17)$ & $\begin{array}{c}(1,2)(2,3)(3,4)(6,7)(7,8)(10,11)(11,12)(12,13) \\
(17,18)(18,19)(19,20)(21,22)(22,23)\end{array}$ & $(19,20)(18,19)$ \\
\hline$(17,18)$ & $\begin{array}{c}(1,2)(2,3)(3,4)(5,6)(6,7)(7,8)(10,11) \\
(11,12)(16,17)(18,19)(19,20)\end{array}$ & $(19,20)$ \\
\hline$(18,19)$ & $\begin{array}{c}(3,4)(5,6)(6,7)(7,8)(10,11)(16,17) \\
(17,18)(19,20)\end{array}$ & Nil \\
\hline$(19,20)$ & $(5,6)(6,7)(7,8)(16,17)(17,18)(18,19)$ & Nil \\
\hline$(21,22)$ & $\begin{array}{c}(1,2)(2,3)(6,7)(7,8)(16,17) \\
(22,23)(23,24)(24,25)\end{array}$ & $(23,24)(24,25)$ \\
\hline$(22,23)$ & $(1,2)(6,7)(7,8)(16,17)(21,22)(23,24)(24,25)$ & $(24,25)$ \\
\hline$(23,24)$ & $(1,2)(7,8)(21,22)(22,23)(24,25)$ & Nil \\
\hline$(24,25)$ & $(21,22)(22,23)(23,24)$ & Nil \\
\hline
\end{tabular}

TABLE IV. OPOCA ChANNEl AsSignMent of Figure 7

\begin{tabular}{|c||c|c|c|}
\hline LINKS & $\begin{array}{c}\text { POC CHANNEL } \\
\text { ASSIGNMENT }\end{array}$ & LINKS & $\begin{array}{c}\text { POC CHANNEL } \\
\text { ASSIGNMENT }\end{array}$ \\
\hline$(1,2)$ & 11 & $(13,14)$ & 6 \\
\hline$(2,3)$ & 1 & $(14,15)$ & 10 \\
\hline$(3,4)$ & 6 & $(16,17)$ & 1 \\
\hline$(5,6)$ & 3 & $(17,18)$ & 1 \\
\hline$(6,7)$ & 9 & $(18,19)$ & 11 \\
\hline$(7,8)$ & 8 & $(19,20)$ & 1 \\
\hline$(9,10)$ & 1 & $(21,22)$ & 1 \\
\hline$(10,11)$ & 4 & $(22,23)$ & 1 \\
\hline$(11,12)$ & 6 & $(23,24)$ & 4 \\
\hline$(12,13$ & ) 7 & $(24,25)$ & 1 \\
\hline
\end{tabular}

to a link $(2,3)$. Same as the case with $\mathrm{xx}[5,6,3]$ shows that the channel 3 is allotted to link $(5,6)$ in a network. The total numbers of partially overlapping channels are 11 , So the value of $\mathrm{xx}$ will proceed to 11 . Similarly for channel 11 this concept will be used, the variable $\mathrm{xx}[10,11,4]$ shows that 4 channel is allocated to the link $(10,11)$ in WMN. The traffic flow is taken as $0.2,0.4,0.8,1.0$ and it exceeds to the value 2.0. These outcomes are taken further for all the demands changing from 50 to 500 packet/ sec. Table IV is completely based on Fig. 7 . Table IV consists of two columns. The first column comprises considered links and second column comsists of POC channles that are assigned to each link.

\section{Information Asymmetry Minimization (IAMin) in AMPL}

In the next step orthogonal channels have been assigned to the same topology for the purpose of comparison of both nonoverlapping and partially overlapping channels. The results of channel assignment for partially overlapping channels and non-overlapping has been compared. Table $\mathrm{V}$ shows channel assignment result for orthogonal channel that are executed through AMPL. The entire orthogonal channel 1, 6, 11 have been assigned to the links. The snapshots have been taken 
TABLE V. NON-OverlapPing ChANNEL ASSignMENT FOR IAMin MODEL

\begin{tabular}{|c|c|}
\hline Links & Non-Overlapping Channel Assignment \\
\hline$(1,2)$ & 11 \\
\hline$(2,3)$ & 1 \\
\hline$(3,4)$ & 6 \\
\hline$(5,6)$ & 1 \\
\hline$(6,7)$ & 1 \\
\hline$(7,8)$ & 11 \\
\hline$(9,10)$ & 1 \\
\hline$(10,11)$ & 1 \\
\hline$(11,12)$ & 1 \\
\hline$(12,13)$ & 11 \\
\hline$(13,14)$ & 1 \\
\hline$(14,15)$ & 1 \\
\hline$(16,17)$ & 1 \\
\hline$(17,18)$ & 1 \\
\hline$(18,19)$ & 11 \\
\hline$(19,20)$ & 1 \\
\hline$(21,22)$ & 1 \\
\hline$(22,23)$ & 1 \\
\hline$(23,24)$ & 1 \\
\hline$(24,25)$ & 11 \\
\hline & \\
\hline
\end{tabular}

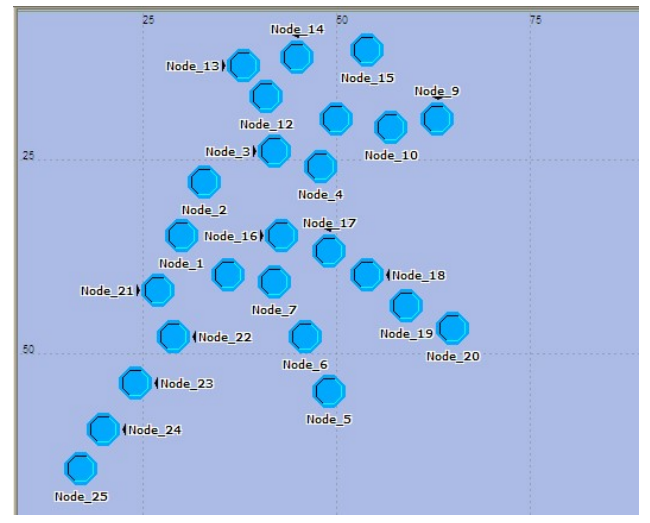

Fig. 12. OPNET generated 25 nodes WMN topology.

during the implementation in AMPL software. The binary variables 0 and 1 are used for orthogonal channel assignment. The binary variable 0 represent no channel has been assigned or absence of channel assignment and 1 represents presence of channel assigned to a link. AMPL assign same traffic load to each link. That is varied from 50 to 500 packets/sec.

Table $\mathrm{V}$ represents the orthogonal channel assignment to each of the link in a network. The channel 1, 6 and 11 have been assigned to the links.

\section{OPNET Simulation Results}

The channel assignment result obtained from POC and nonoverlapping channel assignment through AMPL is further used for simulation process in OPNET. All the three topologies are recreated in OPNET modeler. A network model is created by selecting the area and the location of various nodes in that area. Fig. 12 shows 25 nodes topology that is created with the help of OPNET modeler. Three different topologies each consisting of 25 nodes are constructed and the effect of Information Asymmetry interference in a network have been analyzed for both POC and orthogonal channel assignment.

1) OPNET simulation results for POC model: The channel assignment results obtained from AMPL is used by OPNET simulator for further process. The links have been divided into
TABLE VI. Simulation Parameters For the OPOCA Model

\begin{tabular}{|c||c|}
\hline PARAMETER & VALUE \\
\hline Radio Technology & IEEE $802.11 \mathrm{~b}$ \\
\hline Radio Frequency & $2.4 \mathrm{GHz}$ \\
\hline Data Rate & $11 \mathrm{Mbps}$. \\
\hline Minimum Bandwidth & $22 \mathrm{MHz}$ \\
\hline Buffer size & 25600000 bytes \\
\hline Transmission Range (Tr) & 10 meters \\
\hline Carrier Sensing Range (CSr) & $2 * 10$ meters \\
\hline Number of Nodes & 25 \\
\hline Radios per Node & 3 \\
\hline Network Simulator used & OPNET \\
\hline Simulation time & 4 minutes \\
\hline Terrain area & $80 \times 80 \mathrm{~m} 2$ \\
\hline Total scenarios & 3 \\
\hline
\end{tabular}

TABle VII. Proposed OpOCA Model Simulation Results

\begin{tabular}{|c|c|c|c|c|}
\hline Flow (Packets/Sec) & Topology 1 & Topology 2 & Topology 3 & Average \\
\hline 50 & 924.45 & 1029.59 & 958.17 & 970.736667 \\
\hline 100 & 1720.77 & 1969.27 & 1894.46 & 1861.5 \\
\hline 150 & 1957.53 & 2689.41 & 2663.93 & 2436.95667 \\
\hline 200 & 2061.05 & 3025.85 & 3037.48 & 2708.12667 \\
\hline 250 & 2175.03 & 3404.41 & 3177.13 & 2918.85667 \\
\hline 300 & 2264.11 & 3685.18 & 3324.98 & 3091.42333 \\
\hline 350 & 2355.35 & 3919.37 & 3459.72 & 3244.81333 \\
\hline 400 & 2481.11 & 4133.76 & 3571.12 & 3395.33 \\
\hline 450 & 2577.25 & 4183.24 & 3657.67 & 3472.72 \\
\hline 500 & 2670.51 & 4329.82 & 3828.99 & 3609.77333 \\
\hline
\end{tabular}

coordinated and IA interfering links. The OPNET simulation results are gathered for the proposed model that is Optimized Partial Overlapping Channel Assignment (OPOCA) model. The parameters for simulation are shown in Table VI.

Table VI depicts that the radio technology used for each scenario is IEEE $802.11 \mathrm{~b}$ spectrum. The frequency used is $2.4 \mathrm{GHz}$ and transmission capacity is 11 Mbps. Each node comprises of three radios and different channels are allotted to each radio interface. The demand of flow ranges from 50 to 500 packets $/ \mathrm{sec}$. The total simulation time is four minutes. The results obtained from the simulations shows that the proposed model gives better result as compared to the existing model.

Table VII depicts the simulation results of the effect of IA interference over POC channel assignment. The network capacity of every topology has been measured. The network flow ranges from 50 to 500 (packets/sec) for each topology. The table shows that as the flow demand increases the network capacity value also increases. Minimizing the IA interference increases the overall throughput. The last column shows the average result obtained from different topologies of the OPOCA model. For each topology the network capacity varies depending on the density of a network.

Table VIII represents the results we got from existing IAMin model. The traffic flow varies from 50 to 500 packets per second for each topology. The table shows that as the flow demand increases the network capacity value also increases. Minimizing the IA interference increases the overall throughput. The last column represents average network capacity of the existing model. The transmission capacity of the exiting model is lower than that of the proposed model which clarifies that the OPOCA model works better than IAM model. 
TABLE VIII IAM MODEL SimULATION RESUlTS

\begin{tabular}{|c|c|c|c|c|}
\hline Flow (Packets/Sec) & Topology 1 & Topology 2 & Topology 3 & Average \\
\hline 50 & 979.21 & 1038.79 & 858.25 & 958.75 \\
\hline 100 & 1783.09 & 1840.58 & 1694.1 & 1772.59 \\
\hline 150 & 2239.82 & 2328.93 & 2174.34 & 2247.69667 \\
\hline 200 & 2485.53 & 2619.65 & 2580.12 & 2561.76667 \\
\hline 250 & 2626.37 & 2853.6 & 2836.3 & 2772.09 \\
\hline 300 & 2758.57 & 3037.53 & 3009.57 & 2935.22333 \\
\hline 350 & 2837.99 & 3144.7 & 3235.9 & 3072.86333 \\
\hline 400 & 2935.28 & 3264.71 & 3318.54 & 3172.84333 \\
\hline 450 & 2972.79 & 3369.85 & 3446.81 & 3263.15 \\
\hline 500 & 2982.99 & 3450.61 & 3532.84 & 3322.14667 \\
\hline
\end{tabular}

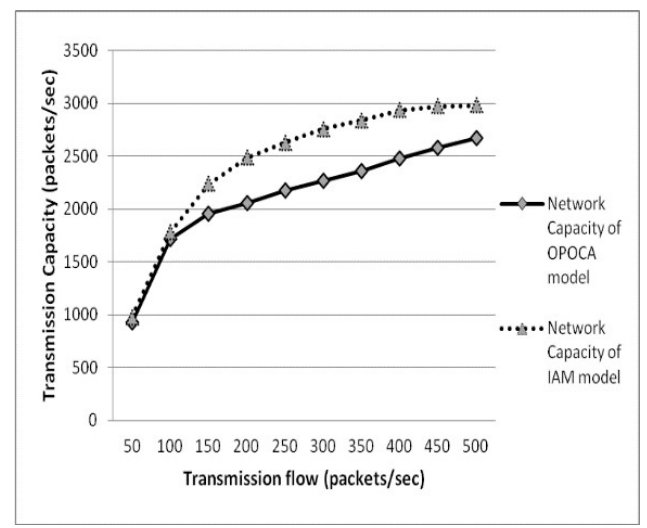

Fig. 13. Capacity comparison of OPOCA model over IAM model for topology 1

Fig. 13 shows the graphical representation of capacity comparison of OPOCA model over the IAMin model. Both the model consists of 25 nodes each. The horizontal axis shows the transmission flow which varies from 50 to 500 packets/sec, whereas the vertical line shows the transmission capacity.

The above dotted line shows the increment in the network capacity of IAMin model and the below solid line represent increase in network capacity of the POC model. From the below graph it has been verified that where the IA interference is minimum the channel assignment of orthogonal channel (IAM) works better than the partially overlapping channel assignment. The network capacity in sparse network of IAM model is maximum than the OPOCA model, where the nodes are aligned far from each other.

Fig. 14 illustrates the capacity comparison of OPOCA model over the existing model. The graph values have been taken from Tables VII and VIII, based Topology 2. Here an $\mathrm{x}$-axis displays sender node demand on every node, whereas $\mathrm{y}$-axis displays the WMN capacity of network in packets/sec. The above solid line represent network capacity of OPOCA model and below dotted line represents the network capacity of IAMin model. As the flow demand increases the network capacity also increases. The peak value for OPOCA model is 4329.82 and the peak value of existing model (IAM) is 3450.61 , from which it is verified that partially overlapping channel assignment works better than IAMin model. In dense network where IA interference is more the partially overlapping channel assignment gives better performance.

Fig. 15 indicate the network capacity relationship among the OPOCA and IAMin model for third topology. For the proposed model the throughput value ranges from $958.17 \mathrm{kbps}$ for 50 packets/sec and increases to $3828.99 \mathrm{kbps}$ for 500

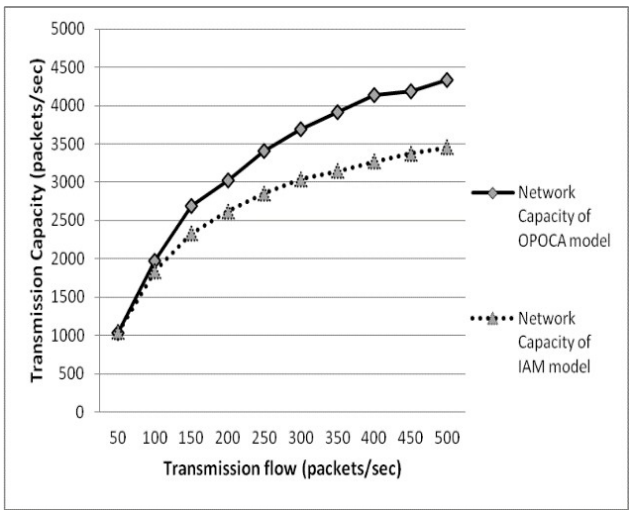

Fig. 14. Capacity comparison of OPOCA model over IAM model for topology 2 .

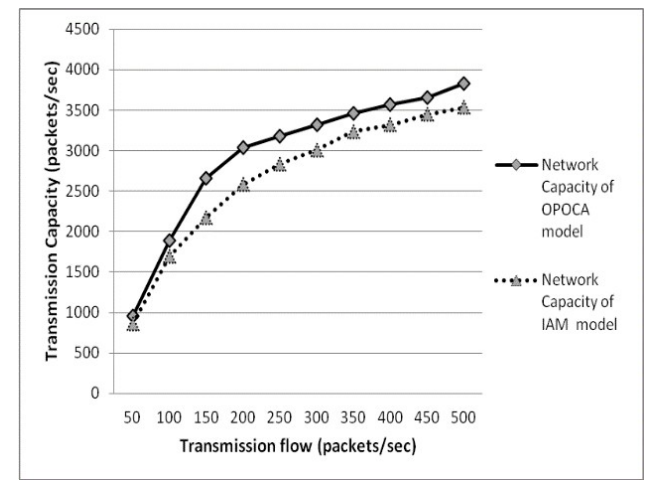

Fig. 15. Capacity comparison of OPOCA model over IAMin model for topology 3 .

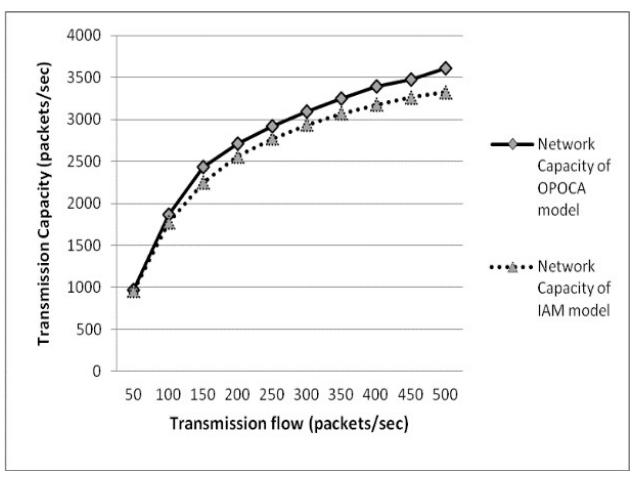

Fig. 16. Capacity comparison of OPOCA model over IAMin model for topology 4 .

packets/sec.

As the traffic flow increases capacity also increases. The IAMin model value ranges from $858.25 \mathrm{kbps}$ for 50 packets/sec and increases to $3532.84 \mathrm{kbps}$ for 500 packets/sec. The graphical representation of data shows that the network capacity of OPOCA model is more than that of IAMin model.

Fig. 16 represents average network comparison of capacity between OPOCA model and IAM model. The upper solid line in graph represents average capacity of an OPOCA model and the lower dotted line shows the average capacity of IAMin model. The horizontal value shows traffic demand in packet/ 
TABLE IX. NetWork CAPACITY IMPROVEMENT OF OPOCA MOdEL OVER IAMIN MODEL

\begin{tabular}{|c|c|c|}
\hline Flow (Packets/Sec) & $\begin{array}{c}\text { Avg Network Capacity } \\
\text { Of IAMin Model }\end{array}$ & $\begin{array}{c}\text { Avg Network Capacity } \\
\text { Of OPOCA Model }\end{array}$ \\
\hline 50 & 958.75 & 970.7367 \\
\hline 100 & 1772.59 & 1861.5 \\
\hline 150 & 2247.697 & 2436.957 \\
\hline 200 & 2561.767 & 2708.127 \\
\hline 350 & 2772.09 & 2918.857 \\
\hline 350 & 2935.223 & 3091.423 \\
\hline 400 & 3072.863 & 3244.813 \\
\hline 450 & 3172.843 & 3395.33 \\
\hline 500 & 3263.15 & 3472.72 \\
\hline & 3322.147 & 3609.773 \\
\hline
\end{tabular}

TABLE X. NETWORK CAPACITY IMPROVEMENT OF OPOCA MODEL OVER IAMIN MODEL

\begin{tabular}{|c|c|c|c|}
\hline Flow (Packets/Sec) & $\begin{array}{c}\text { Percentage Capacity } \\
\text { Of IAMin Model }\end{array}$ & $\begin{array}{c}\text { Percentage Capacity } \\
\text { Of OPOCA Model }\end{array}$ & $\begin{array}{c}\text { Percentage (\%) } \\
\text { Improvement }\end{array}$ \\
\hline 50 & 95 & 97 & 2 \\
\hline 100 & 88 & 93 & 5 \\
\hline 150 & 75 & 81 & 6 \\
\hline 200 & 64 & 68 & 4 \\
\hline 250 & 55 & 58 & 3 \\
\hline 300 & 48 & 51 & 3 \\
\hline 350 & 43 & 46 & 3 \\
\hline 400 & 39 & 42 & 3 \\
\hline 450 & 36 & 38 & \\
\hline 500 & 33 & 36 & \\
\hline
\end{tabular}

sec for the topology of 25 nodes. Similarly the vertical line in the graph shows the normal network capacity for those models i.e. consist of existing and proposed model. The graph shows that the average network capacity is improved by POC model, and gives better performance than the IAMin model. As the IA interference is minimized by optimal channel assignment the overall network capacity increases. Tables IX and X represents that the average network capacity of the proposed model that is $62 \%$ and the network capacity of the existing IAM model is $57 \%$. The percentage improvement between existing and proposed model is 5\% that shows, the Optimized Partially Overlapping Channel Assignment (OPOCA) model provides better capacity improvement in a dense environment over the Information Asymmetry Minimization (IAMin) model.

\section{CONClusion}

In this research the information asymmetry interference is minimized and WMN network capability is maximized. The OPOCA model is compared with the IAM model that is without the node radio constraint and partially overlapping channel constraint. The simulation has been carried out on 25 node topology and the result shows that the proposed channel assignment scheme gives better result where the information asymmetry is high, than the existing model.The average network capacity of the proposed model is $63 \%$ and the network capacity of the existing IAMin model is 55\%. The percentage improvement between existing and proposed model is $8 \%$ that shows, the Optimized Partially Overlapping Channel Assignment (OPOCA) model provides better capacity improvement in a dense environment over the Information Asymmetry Minimization (IAM) model. Through simulation results it is verified that OPOCA model provides considerable capacity improvement over the IAMin model.

\section{ACKNOWLEDGMENT}

The authors would like to thank the University of Agriculture Peshawar and FATA university FR, Kohat Pakistan for supporting the research done in this paper. The co-authors also participated enthusiastically and did their full efforts to explore the channel assignment and its results in Wireless mesh networks.

\section{REFERENCES}

[1] Gledson, E., C. Daniel, P. Ferreira, and Gustavo (2011) A Layered Routing Architecture for Infrastructure Wireless Mesh Networks. In Networking and Services, 2009.ICNS'09. Fifth International Conference on, pp. 366-369.

[2] Chen, Y., N. Xie, G. Qian, and H. Wang, (2010). Channel assignment schemes in Wireless Mesh Networks, In: Mobile Congress (GMC), 2010 Global, vol., no., pp.1-5, 18-19 Oct. 2010

[3] Shah, S., H. Hussain, and M. Shoaib, (2013, September). Minimizing non-coordinated interference in multi-radio multi-channel Wireless Mesh Networks (MRMC-WMNs). In Digital Information Management (ICDIM), 2013 Eighth International Conference on, pp. 24-28.IEEE, 2013.

[4] Shah, S., H. Hussain, and M. Shoaib, (2013, September). Minimizing non-coordinated interference in multi-radio multi-channel Wireless Mesh Networks (MRMC-WMNs). In Digital Information Management (ICDIM), 2013 Eighth International Conference on, pp. 24-28.IEEE, 2013.

[5] Shinde, S. R., M. L. Dhore, and J. B. Karande, (2009) Avoiding Interferences in WLAN 802.11b for PartiallyOverlapped Channels.International Conference on Advances in Computing, Communication and Control (ICAC309)

[6] Weisheng, S. (2010) An overview of Channel Assignment methods for multi-radio multi-channel wireless mesh networks Volume 70, Issue 5, Pages 505524.

[7] Pollak, S. and V. Wieser, (2012) Channel Assignment SchemesOptimization for Multi-InterfaceWireless Mesh Networks Based on Link Load. INTECH Open Access Publisher, 2012.

[8] Venkata, K. and M. M. Naidu, (November, 2014) Evaluation of Interference-Aware Channel Allocation Algorithms for Wireless Mesh Networks.International Journal of Emerging Research in Management \& Technology ISSN: 2278-9359 (Volume-3, Issue-11)

[9] Wang, J., W. Shi, K. Cui, F. Jin, and Y. Li, (2015) Partially overlapped channel assignment for multi-channel multi-radio wireless mesh networks. EURASIP Journal on Wireless Communications and Networking (2015)

[10] Garetto, M., T. Salonidis, and E. W. Knightly, (2008). Modeling perflow throughput and capturing starvation in CSMA multi-hop wireless networks. IEEE/ACM Transactions on Networking (TON), 16(4), 864877.

[11] Shah, S., A. W. Abbas, H. Hussain and H. Israr, (2017) Minimizing information asymmetry interference in multi-radio multi-channel wireless mesh networks. Kuwait Journal of Science, 44(3) pp. 30-39.

[12] Shah, S., M. Atif, S. Khan, M. Daud and F. K. Khalil (2018). A Comparison of Near-Hidden and Information Asymmetry Interference Problems in Wireless Mesh Networks. (IJACSA) International Journal of Advanced Computer Science and Applications, Vol. 9, No. 4, 2018 